\title{
Characteristics of vortex packets in turbulent boundary layers
}

\author{
By BHARATHRAM GANAPATHISUBRAMANI, \\ ELLEN K. LONGMIRE AND IVAN MARUSIC $†$ \\ Department of Aerospace Engineering and Mechanics, University of Minnesota, \\ 107 Akerman Hall, 110 Union Street SE, Minneapolis, MN 55455, USA
}

(Received 8 August 2002 and in revised form 25 October 2002)

Stereoscopic particle image velocimetry (PIV) was used to measure all three instantaneous components of the velocity field in streamwise-spanwise planes of a turbulent boundary layer at $R e_{\tau}=1060\left(R e_{\theta}=2500\right)$. Datasets were obtained in the logarithmic layer and beyond. The vector fields in the $\log$ layer $\left(z^{+}=92\right.$ and 150) revealed signatures of vortex packets similar to those proposed by Adrian and co-workers in their PIV experiments. Groups of legs of hairpin vortices appeared to be coherently arranged in the streamwise direction. These regions also generated substantial Reynolds shear stress, sometimes as high as 40 times $-\overline{u w}$. A feature extraction algorithm was developed to automate the identification and characterization of these packets of hairpin vortices. Identified patches contributed $28 \%$ to $-\overline{u w}$ while occupying only $4 \%$ of the total area at $z^{+}=92$. At $z^{+}=150$, these patches occupied $4.5 \%$ of the total area while contributing $25 \%$ to $-\overline{u w}$. Beyond the $\log$ layer $\left(z^{+}=198\right.$ and 530$)$, the spatial organization into packets is seen to break down.

\section{Introduction}

The structure of the turbulent boundary layer has been the subject of much research over the past 60 years because of its importance in practical applications. Some of the current studies are aimed at developing models to represent the boundary layer efficiently at higher Reynolds numbers. Various models have been proposed for the dominant structure, including long quasi-streamwise vortices in the viscous buffer region (see Lyons, Hanratty \& McLaughlin 1989 and Heist, Hanratty \& Na 2000). However, a growing consensus is emerging for a model based on individual hairpin vortices (Theodorsen 1952) which is probably the simplest structural model that explains most of the features observed in wall turbulence. The existence of asymmetric hairpins in the region above the viscous layer was documented by Robinson (1991) in his analysis of early direct numerical simulation (DNS) results. Earlier, Offen \& Kline (1975) used a hairpin model to explain the existence of ejections (Q2 events, $u<0, w>0$ ), volumes of low-speed fluid that are pushed away from the wall, and sweeps (Q4 events, $u>0, w<0$ ), volumes of faster fluid pushed towards the wall, in the logarithmic part of the boundary layer. In this paper $u, v$ and $w$ are defined as the fluctuating velocity components in the streamwise $(x)$, spanwise $(y)$ and wall-normal (z) directions respectively. Willmarth \& Lu (1971) and Blackwelder \& Kaplan (1976), among others, documented that ejections and sweeps make a substantial contribution 
to the Reynolds shear stress and therefore to the drag associated with the bounding surface.

Bandyopadhyay (1980) and Head \& Bandyopadhyay (1981) proposed that hairpin vortices travel in groups, from the flow visualization experiments they performed on a zero-pressure-gradient boundary layer. Theoretical studies by Smith et al. (1991) and the study of DNS datasets at low Reynolds number $\left(R e_{\tau}=\delta U_{\tau} / v=180\right.$, where $\delta$ is the boundary layer thickness, $U_{\tau}$ is the skin friction velocity and $v$ is the kinematic viscosity) performed by Zhou et al. (1999) demonstrated that a single vortex of sufficient circulation could spawn a trailing group of hairpins that was convected at the same speed as the leading structure. More recently, Adrian, Meinhart \& Tomkins (2000b), who performed particle image velocimetry (PIV) experiments in $(x, z)$-planes of a zero-pressure-gradient boundary layer over a Reynolds number range of $355<R e_{\tau}<2000$, identified packets of hairpin vortex heads that appeared regularly in the near-wall and logarithmic layers. Groups of 5-10 hairpins, which extended over a length of $2 \delta$, were found to convect at a uniform streamwise velocity. The authors noted also that the presence of vortex packets explained the multiple ejections associated with individual bursts observed by Bogard \& Tiederman (1986). The elongated zones of uniform streamwise velocity associated with the packets also help explain the long tails in two-point correlations of streamwise velocity found in Kovasznay, Kibens \& Blackwelder (1970), Townsend (1976) and Brown \& Thomas (1977). Recently, Christensen \& Adrian (2001), with PIV data in $(x, z)$ planes of channel flow at $R e_{\tau}=547$ and 1734, used linear stochastic estimation for the conditionally averaged velocity field associated with swirling motion. They concluded that the mean structure consists of a series of swirling motions along a line inclined at $12^{\circ}-13^{\circ}$ with the wall, which is consistent with the earlier observations of packets of hairpin vortices. An attached-eddy model applied by Marusic (2001) also demonstrated that packets of eddies were required in order to match measured boundary layer turbulence statistics near the wall.

In recent work similar to the present study (Tomkins \& Adrian 2002, see also the thesis by Tomkins 2001), PIV measurements of streamwise-spanwise planes of a turbulent boundary layer at $430<R e_{\tau}<2270$ (only in-plane velocity components were measured) showed long low-streamwise-momentum zones enveloped by positive and negative vortex cores representing packets of hairpin structures.

The objective of the current study is to apply the stereo PIV technique to examine $(x, y)$-planes in the outer region of a zero-pressure-gradient boundary layer $\left(z^{+}=z U_{\tau} / v>60\right)$ in order to identify the typical structures and to determine their contribution to the Reynolds shear stress, thereby investigating the role, if any, the packet structures play in the transport of momentum in the boundary layers. Based on the previous work described above, feature identification algorithms were developed to search for individual hairpin vortices as well as packets of hairpins.

\section{Facility and methods}

\subsection{Experimental facility, methods and qualification}

The measurements were carried out in a suction wind tunnel with working section of $0.33 \mathrm{~m}$ height, $1.22 \mathrm{~m}$ width and $4.8 \mathrm{~m}$ length. The coordinate system used is depicted in figure 1. Measurement planes were located $3.3 \mathrm{~m}$ downstream of a trip wire in a zero-pressure-gradient flow with free-stream velocity $U_{\infty}=5.9 \mathrm{~m} \mathrm{~s}^{-1}$. Hot-wire measurements showed that the turbulence intensity in the free stream was less than $0.2 \%$. The wall shear stress $\left(\tau_{w}\right)$ was computed using the mean velocity profile and the 


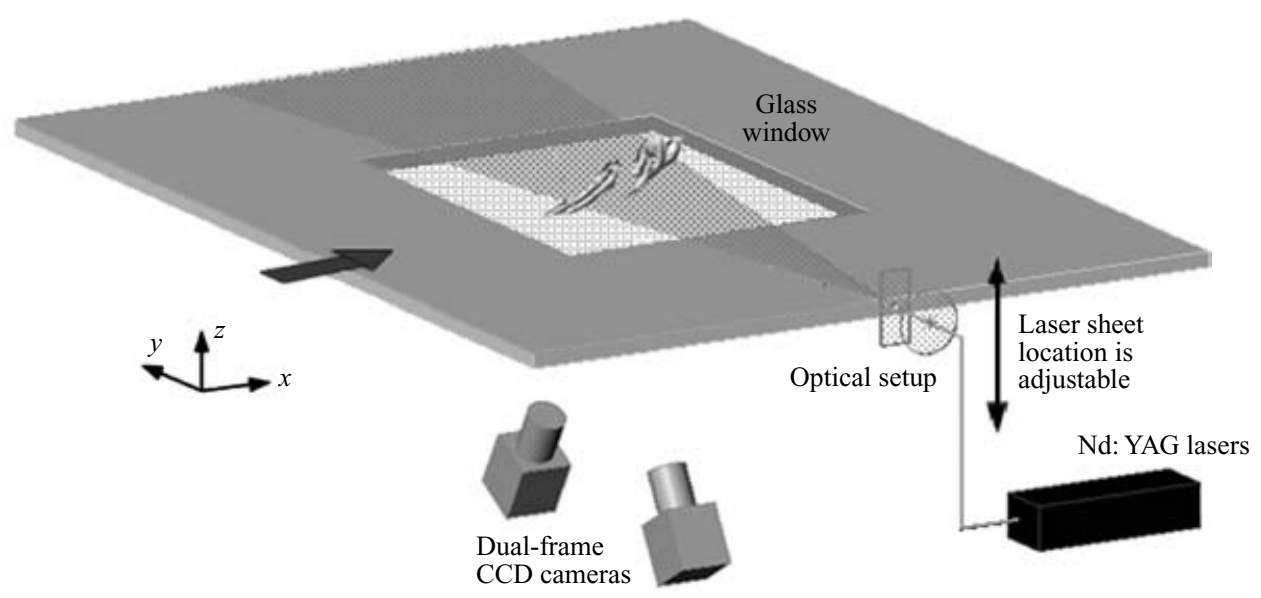

FIGURE 1. Experimental facility.

Clauser chart method. All quantities measured and computed are normalized using the skin friction velocity $U_{\tau}\left(=\sqrt{\tau_{w} / \rho}\right.$, where $\rho$ is the density of the fluid) and $\nu$, and are denoted with a superscript + . The Reynolds number based on the momentum thickness $R e_{\theta}$ was 2500, and $R e_{\tau}$ was 1060 . The value of $\delta$ in the region of the measurement planes was $69 \mathrm{~mm}$.

The flow was seeded with olive oil droplets (size $\sim 1 \mu \mathrm{m}$ ) that were generated by eight Laskin nozzle units set up in parallel. The oil droplets were ingested into the intake of the wind tunnel upstream of honeycomb straighteners and screens used for flow conditioning. At the test section, glass sidewalls and a glass bottom wall were installed in the wind tunnel to provide high-quality optical access. The seed particles were illuminated by pulsed sheets from two Nd:YAG lasers (Big Sky CFR200) directed through one side window and oriented parallel with the bottom wall of the tunnel (see figure 1). The laser pulse energy was $120 \mathrm{~mJ}$, and the thickness of each sheet was $0.3 \mathrm{~mm}$. Sets of digital images were captured by two Kodak Megaplus CCD cameras $(1024 \times 1024$ pixels $)$ at $z^{+}=92,198(z / \delta=0.2)$ and $530(z / \delta=0.5)$ and by TSI Powerview $2048 \times 2048$ pixel resolution cameras at $z^{+}=150$. Nikon Micro Nikkor $60 \mathrm{~mm} \mathrm{f} / 2.8$ lenses were used with both camera types. A TSI synchronizer box controlled the strobing and timing of the cameras and lasers. The dual frame acquisition rate was $15 \mathrm{~Hz}$. The cameras were aligned in a plane parallel with the $(x, y)$ flow plane and inclined at angles of $15^{\circ}$ with the $z$-axis as shown in figure 1 . Additional details of the calibration, experimental arrangement and vector reconstruction schemes are given in Ganapathisubramani, Longmire \& Marusic (2002).

For all derived quantities, the interrogation spot size used was $16 \times 16$ pixels $(\sim 20 \times 20$ wall units) with $50 \%$ overlap. Hence the spacing between adjacent vectors in either direction is 10 wall units $(\sim 0.65 \mathrm{~mm})$. The measurements had two main sources of uncertainty. First, the Gaussian peak fit in the cross-correlation algorithm generated an uncertainty of approximately 0.1 pixels or $0.016 \bar{U}$ (where $\bar{U}$ is the mean velocity in the streamwise direction). Second, a residual error arose due to the leastsquare curve-fit in solving the four pixel-displacement equations in three unknowns (see Ganapathisubramani et al. 2002 for details). This residual error can play a major role in the uncertainty if concentration gradients in seeding occur within the flow field. In this experiment, such gradients were not significant, and this error was on average 


$\begin{array}{rccccccc}z^{+} & N_{t} & \bar{U}^{+} & \overline{u^{2}} & \overline{v^{2}} & \overline{w^{2}} & -\overline{u w^{+}} & \sigma_{\omega_{z}}^{+} \\ 92 & 750 & 16.3 & 2.96 & 1.25 & 1.28 & 0.91 & 0.0422 \\ 150 & 300 & 17.1 & 2.85 & 1.31 & 1.14 & 0.78 & 0.0378 \\ 198 & 750 & 18.7 & 2.78 & 1.06 & 1.32 & 0.83 & 0.0334 \\ 530 & 750 & 21.5 & 1.46 & 0.99 & 1.31 & 0.49 & 0.0212\end{array}$

TABLE 1. Ensemble-averaged flow statistics. $\sigma_{\omega_{z}}$ is the r.m.s (root mean square) of the fluctuating wall-normal vorticity and $N_{t}$ is the total number of PIV realizations included in the ensemble.

about half of the Gaussian error. The vector fields from the images acquired with the Megaplus cameras covered an area of $1.2 \delta \times 1.2 \delta$. For the Powerview cameras, the field size was approximately $2.4 \delta \times 2.4 \delta$ making the spacing between vectors similar to that in the other planes. The larger area was acquired by moving the plane of the cameras away from the object plane.

Velocity gradients were computed from vector fields using a second-order central difference scheme wherever possible in the domain and a first-order forward or backward difference at the boundaries. To identify swirling motion caused by the eddies in the flow field, we used swirl strength $\lambda_{c i}$ (Zhou et al. 1999), which is defined as the magnitude of the imaginary part of the eigenvalue of the local velocity gradient tensor. Since the PIV images were planar, we used only the in-plane gradients and formed a two-dimensional tensor. Vortices could be identified by extracting iso-regions of $\lambda_{c i}$ (Adrian, Christensen \& Liu 2000a).

The values of $\bar{U}$ computed from the PIV data at each wall-normal location agreed well with the hot-wire measurements. Table 1 shows a summary of some ensembleaveraged turbulence statistics. The values shown compare well with previous data in the literature (Balint, Wallace \& Vukoslavcevic 1991; Naguib \& Wark 1992; Adrian et al. 2000b).

\subsection{Feature extraction}

Although a variety of coherent structures can be found in a turbulent boundary layer, we focus on the hairpin vortices discussed in the introduction. Since, the stereo PIV data are obtained in streamwise-spanwise planes, hairpin legs could appear as neighbouring cores of positive and negative wall-normal vorticity $\left(\omega_{z}\right)$ aligned in the spanwise direction. If these hairpins were to travel in groups, one might also see multiple pairs of cores aligned approximately along the streamwise direction. Also, individual or groups of angled hairpins are expected to generate large values of instantaneous Reynolds shear stress $\left(-u w^{+}=-u w / U_{\tau}^{2}\right)$. Hence, an algorithm was developed to identify regions in which all of these events occur. In the algorithm description, the flow is assumed to be from left to right:

Step 1: Regions of positive vorticity $>1.5 \sigma_{\omega_{z}}^{+}$and negative vorticity $<-1.5 \sigma_{\omega_{z}}^{+}$are detected. At $z^{+}=92$, this value was \pm 0.065 .

Step 2: Zones of strong Reynolds shear stress $\left(-u w^{+}\right)$greater than $2 \sigma_{-u w}^{+}$(where $\sigma_{-u w}^{+}$is the r.m.s of $\left.-u w^{+}\right)$are detected independently. No distinction is made between sweep or ejection events. The values of $\sigma_{-u w}^{+}$at $z^{+}=92$ and 150 were 3.43 and 2.68 respectively.

Step 3: All the zones found in step 2 are searched to mark regions of high Reynolds shear stress occurring between strips of positive and negative vorticity (found in step 1) 
such that positive vorticity lies beneath negative vorticity. This vorticity arrangement was chosen to fit the pattern displayed by the legs of a hairpin vortex.

Step 4: Each location of large $-u w^{+}$marked in step 3 is used as a seed point for a region-growing algorithm. This algorithm, based on the streamwise velocity, identifies a connected region of neighbouring points lying within local thresholds such that the final coherent region is a patch of uniform momentum. The lower bound of the velocity was fixed as the minimum velocity in the Reynolds shear stress zone and the upper bound was the average velocity in the two surrounding vorticity regions. If multiple seed zones are enveloped by the same vorticity regions, the thresholds are adjusted such that the lower bound is the minimum velocity at all the seed points of $-u w^{+}$in that region. If the region growth from one location intersects the patches from other seed points, then they are merged and marked as one patch.

Step 5: After detection of all patches in a given vector field, patches that are adjacent to one another in the streamwise direction are merged provided that the streamwise distance between the patches is less than the average width of the patches being merged.

\section{Results and discussion}

Note that the flow is from left to right in all vector plots, and the local mean $\bar{U}$ is subtracted from the vectors to clearly illustrate the slow- and fast-moving zones. The contour/vector plots shown in figure 2 are from individual realizations, but the patterns are representative of those found in many vector fields. The plots revealing the statistical features of the hairpin packets (figure 3) were obtained by investigating all vector fields at a given wall-normal position.

\subsection{Hairpin packets}

Examples of packets found by the feature extraction algorithm at $z^{+}=92$ and 150 are shown in figure 2. Plots of instantaneous vorticity $\left(\omega_{z}^{+}\right)$and Reynolds shear stress $\left(-u w^{+}\right)$at $z^{+}=92$ are shown in figures $2(a)$ and $2(b)$. The $\omega_{z}^{+}$plot clearly reveals a pattern of cores of opposite sign of $\omega_{z}^{+}$organized along the streamwise direction that is indicative of hairpin legs intersecting the measurement plane. Contours of $\lambda_{c i}^{+}$(not shown) were also studied and confirmed the presence of swirling motion corresponding to the centres of blobs of vorticity. The region outlined in black (A) is the zone of uniform momentum identified by the extraction algorithm. The presence of this zone sandwiched between the cores of positive and negative vorticity confirms the claim that the hairpin vortices convect with similar speeds as part of a group. The Reynolds shear stress plot contains values as large as $20 U_{\tau}^{2}$ in the zone of uniform momentum between the vortex cores. Further, the negative direction of the vector arrows in the patch in combination with positive $-u w^{+}$values indicates the presence of a Q2 event where slow-moving fluid is ejected away from the wall. The vorticity plot reveals at least five pairs of vortex cores aligned in the streamwise direction, and the $-u w^{+}$plot shows five distinct regions of strong $-u w^{+}$just upstream of a pair. These regions are probably caused by hairpins inclined at an angle to the wall that eject fluid upward between their legs. Finally, note that the packet extends all the way across the field $(\sim 1.2 \delta)$. Additional plots of $-u w^{+}$(not shown) revealed values as high as $40 U_{\tau}^{2}$ within packets compared with the mean value of only $0.91 U_{\tau}^{2}$. Plots of complete fields depicting vorticity, swirl strength and Reynolds shear stress within and surrounding packets can be found in Longmire, Ganapathisubramani \& Marusic (2001). 


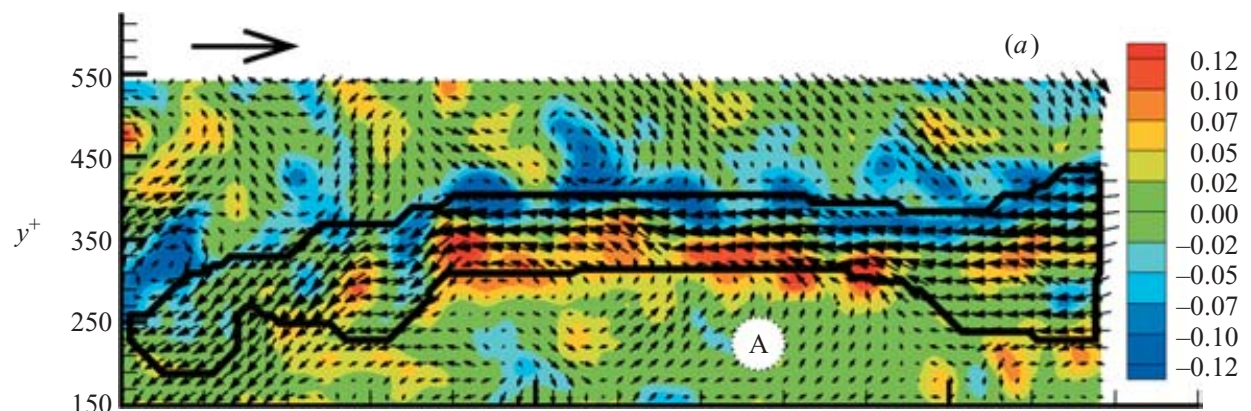

(b)
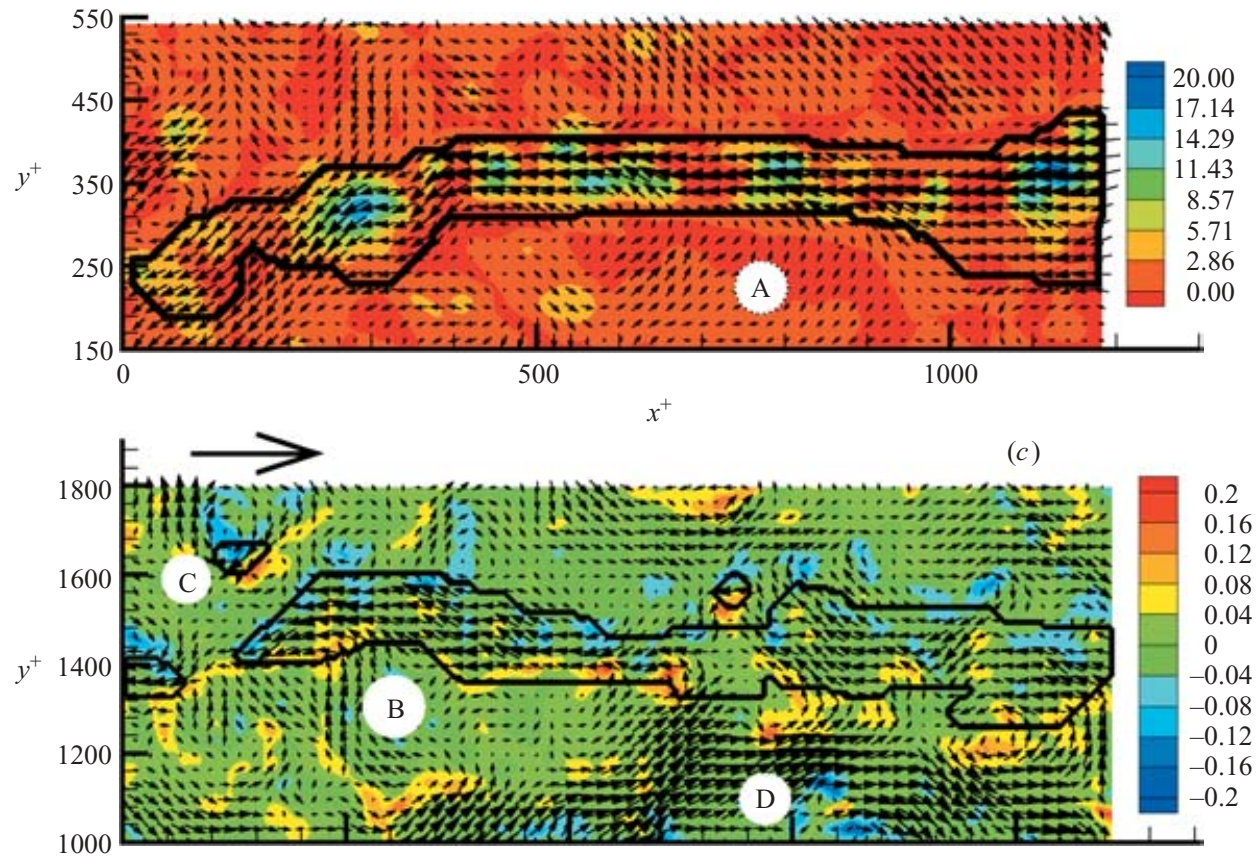

(d)

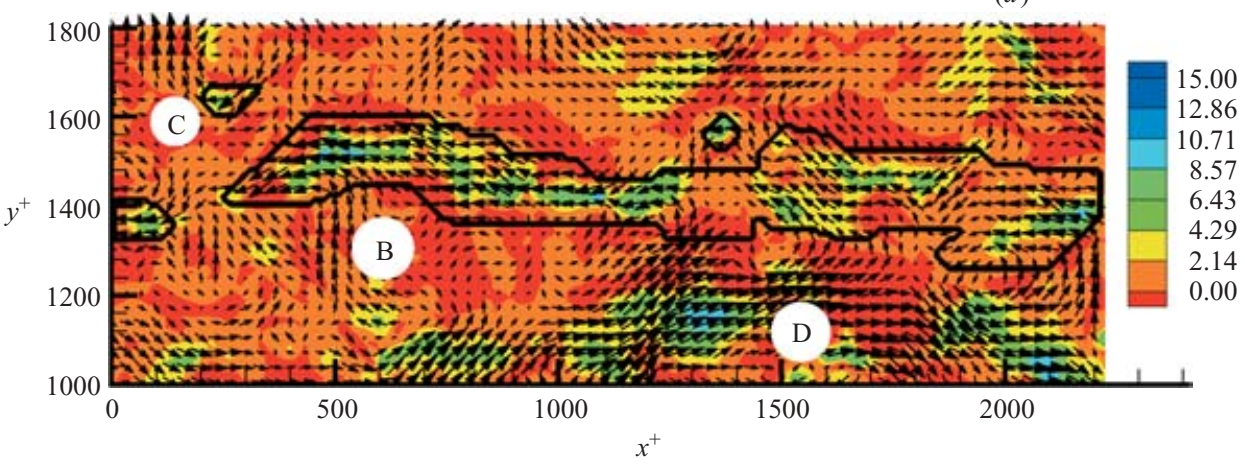

FIGURE 2. Hairpin packets identified using the feature extraction algorithm. At $z^{+}=92$, for clarity only every second vector resolved is shown: $(a) \omega_{z}^{+},(b)-u w^{+}$. At $z^{+}=150$, every third vector resolved is shown: $(c) \omega_{z}^{+},(d)-u w^{+}$. Note that $(c)$ and $(d)$ cover four times the area of $(a)$ and $(b)$. The flow is from left to right and the vectors shown have $\bar{U}$ subtracted. 
Usually, the hairpin vortices observed have two legs. The legs may be aligned or somewhat offset in streamwise location. Sometimes, the hairpins appear to have single legs or have significant asymmetries in the strength of each leg (see the single negative vortex core to the left of region marked A at $x^{+}=50$ and $y^{+}=280$ in figure $2 a$ ). Although Robinson (1991) saw mostly single-legged hairpins in DNS datasets at low Reynolds numbers, Tomkins \& Adrian (2002) in their PIV measurements of $(x, y)$ planes at higher Reynolds numbers found both symmetric and asymmetric structures.

Figures $2(c)$ and $2(d)$ are larger views with plots of $\omega_{z}^{+}$and $-u w^{+}$at $z^{+}=150$. The total field length is twice as long as in figures $2(a)$ and $2(b)$. The region marked $\mathrm{B}$ is a zone of uniform momentum identified by our feature extraction algorithm. The contours of $\omega_{z}^{+}$and $\lambda_{c i}^{+}$(not shown) along with the vectors again show regions of swirling motion bounding the patch. The Reynolds shear stress shows discrete zones of high magnitude along the entire length of $\mathrm{B}$. The zones of strong $-u w^{+}$ always seem to occur between pairs of swirls on opposite sides of the patch. In this patch, the maximum values of $-u w^{+}$are smaller than in figure $2(b)$, but they are still significantly higher than the global mean $\left(-\overline{u w} \bar{w}^{+}=0.78\right)$. The length of the patch is $1.9 \delta$.

Many shorter patches like $\mathrm{C}$ were also identified at both $z^{+}=92$ and 150 . These patches usually contained either one or two vortex core pairs and a small area contributing to the Reynolds shear stress. There are also even smaller patches like the one just above patch (B). On further investigation of the $\lambda_{c i}^{+}$plot, it is clear that this is a single hairpin vortex encompassing a small area of significant $-u w^{+}$. However, it is unclear whether this or other smaller patches travel with nearby longer patches. Sometimes, patches containing $2-3$ hairpins were closely followed by an additional short patch at a streamwise distance of 150-300 wall units.

Region D exhibits Q4 characteristics. From the direction of the vector arrows it is quite clear that $u$ is greater than zero and $-u w^{+}$is positive, so $w$ must be less than zero. These regions frequently appeared adjacent to packets identified by the algorithm at both $z^{+}=92$ and 150 . The inclination of hairpin vortices with respect to the wall is such that the region outside of the legs/necks should induce Q4 events. These Q4 regions often carry a significant amount of Reynolds shear stress, and the maximum local values are comparable to the maximum values observed in a Q2-type event $\left(-u w^{+} \sim 40\right)$.

The patches identified by the algorithm are typically aligned in the streamwise direction but sometimes inclined at small angles such that they have a spanwise component. The series of vortex cores are at times offset in the spanwise direction by 100-150 wall units so that the zones of uniform momentum are oriented at an angle of $10^{\circ}-20^{\circ}$ to the streamwise direction.

The data acquired further from the wall $\left(z^{+}=198\right.$ and 530) revealed a variety of coherent structures, but no patterns of long low-speed streaks, elongated regions of high Reynolds shear stress or organization of vorticity in the form of a train of hairpins. Thus, the results indicate that the legs of most hairpins do not extend as far as $z / \delta=0.2\left(z^{+}=198\right)$. However, it is possible that the largest hairpin in a given packet crosses this measurement plane such that only individual structures are evident. Typical structures observed included individual vortex cores, large regions of upwash with scales approaching 300 wall units, and spanwise strips of wall-normal velocity with alternating signs, possibly indicative of hairpin heads. Contour plots of wall-normal vorticity and velocity at $z^{+}=198$ are shown in Longmire et al. (2001). At $z / \delta=0.5\left(z^{+}=530\right)$, we observed only occasional large vortex cores with diameter of up to 300 wall units. 

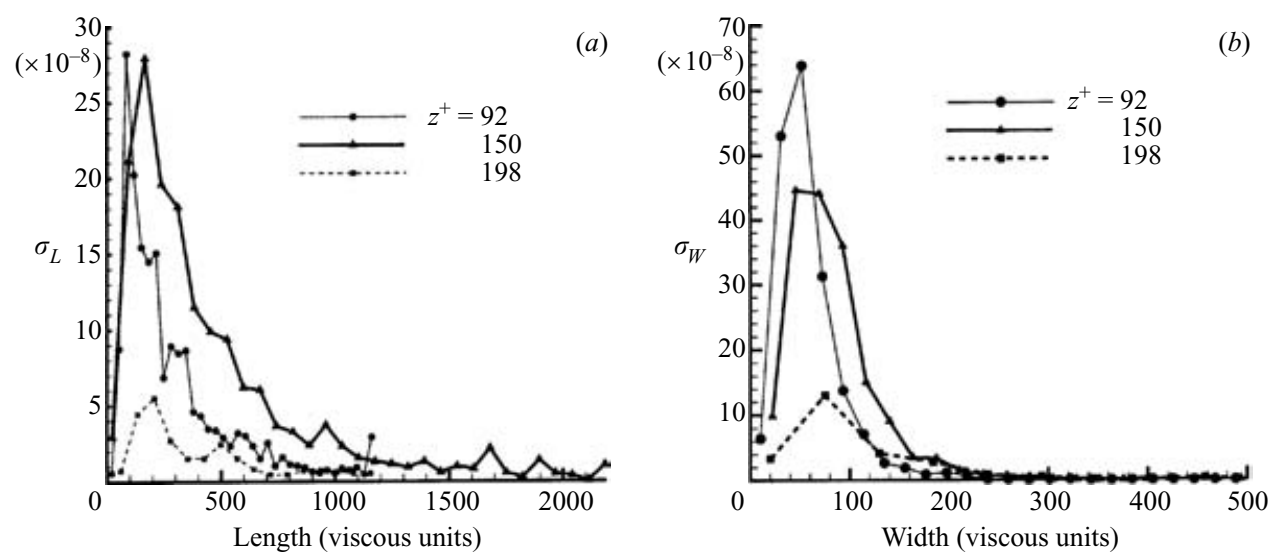

FIGURE 3. Length and width statistics of packets found by the feature extraction algorithm. The total number of patches identified $\left(N_{p}\right)$ at $z^{+}=92,150$ and 198 are 1940, 3040 and 380 respectively.

The reduced spatial coherence in the streamwise direction at the edge of the logarithmic layer and beyond is consistent with the results obtained by Adrian et al. $(2000 b)$ in their PIV measurements of streamwise-wall-normal planes. This behaviour is also consistent with the attached-eddy modelling study by Marusic (2001), in which the kinematic state of the boundary layer was modelled by an ensemble of eddies of varying height. The results of that study suggested that packets with long streamwise coherence did not need to extend beyond the log layer in order to obtain the appropriate correlation statistics. In this region, only uncorrelated eddies were required.

Figures $3(a)$ and $3(b)$ show the length and width distributions of the patches identified by the feature extraction algorithm in all vector fields at $z^{+}=92,150$ and 198. The ordinate is the number of patches found in a certain bin divided by the total area searched in all of the vector fields. The length distribution clearly illustrates the lack of patches at $z^{+}=198$ relative to the two planes in the logarithmic layer. The peak of this distribution in all three planes, which occurs near 100 wall units, coincides with the identification of single vortex cores. The long tail of the distribution at $z^{+}=150$ (where measurement planes had the largest dimension) reveals a significant number of packets with length on the order of $2 \delta$. This result is supported by the findings of Tomkins \& Adrian (2002) whose PIV datasets revealed zones of uniform momentum stretching as far as $2.5 \delta$. Much larger measurement planes (difficult to achieve with the available illumination energy) would be required to determine the maximum length of the patches. The width distributions in figure $3(b)$ peak from 50 to 80 wall units, and do not exhibit long tails. The shape of these distributions indicates that the patches are typically narrow in the spanwise direction. The length and width results are consistent with two-point correlations and autocorrelations of the streamwise velocity found in the literature (e.g. Townsend 1976, p. 112) which indicate that the zones of uniform momentum extend to a length of order $3 \delta$ in the streamwise direction.

Although the width distribution found by the feature detection algorithm appears accurate, the length distribution might change with a more sophisticated algorithm. In many cases, individual short patches were found to be separated by distances of about 100 wall units in the streamwise direction, and these patches were not merged by the 

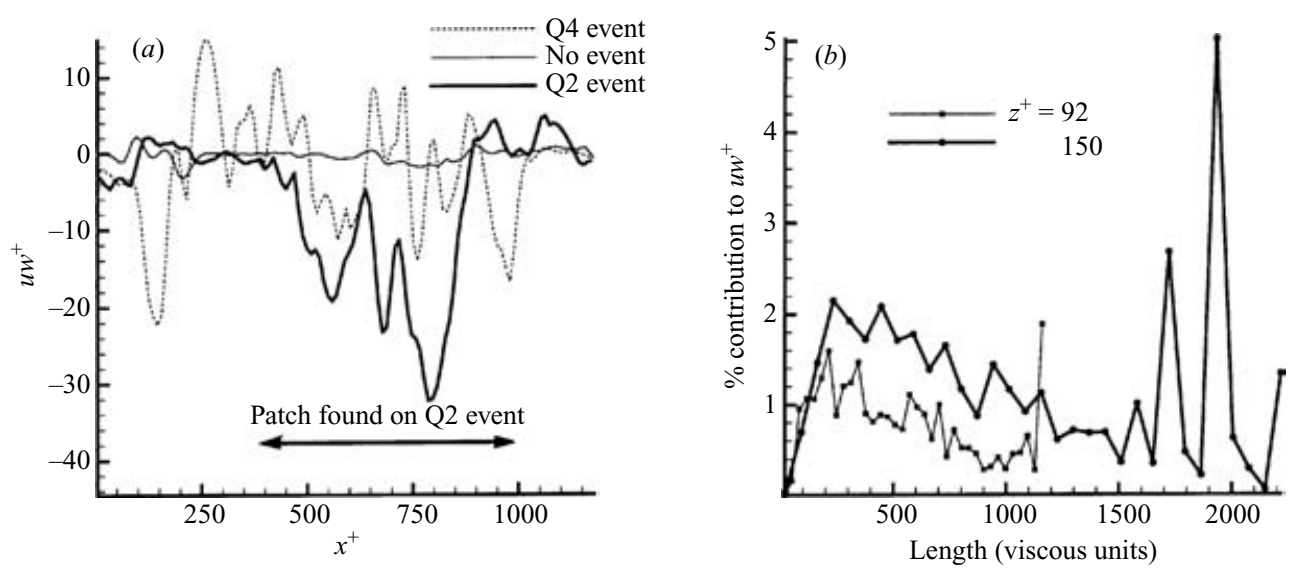

FIGURE 4. (a) Typical $u w^{+}$signature showing different events at $z^{+}=92 ;(b) \%$ contribution to $\overline{u w}{ }^{+}$. Measuring field of view length for $z^{+}=150$ was twice that used for $z^{+}=92$.

algorithm. From examination of individual fields, we suspect that these short patches in reality could be part of longer patches with length of order $\delta$. Thus, with improved identification techniques, the length distribution curve would probably become flatter such that the peak near 100 viscous units would decrease in value while the number of patches with greater length would increase.

\subsection{Reynolds shear stress contributions}

In figure 4(a), values of $u w$ have been extracted along streamwise lines of fields at $z^{+}=92$ for a typical instantaneous realization. The curve for Q2 shows $u w$ including the approximate centerline of an identified patch where the extent of the patch is marked. The values of $-u w$ are large through most of the patch and reach a maximum near $35 U_{\tau}^{2}$. This behaviour can be compared with a line taken through a short sweep (Q4). The feature extraction algorithm does not pick out these regions because the combination of vorticity sought finds zones with negative $u$ only. The Q4 line fluctuates fairly rapidly between positive and negative values of $u w$ of significant magnitude. The maximum magnitude of $20 U_{\tau}^{2}$ is associated with a short negative peak. A line along a zone not including any packet or obvious strong event is also shown. In this case, the curve oscillates around zero at very small magnitudes. This behaviour, in fact, occurs along many lines in the PIV plots, which is not surprising considering the shape of the probability density function of $u w$ found in Willmarth \& $\mathrm{Lu}$ (1971). The ratio of the total Reynolds shear stress from Q2 areas to that of Q4 areas was about 1.1. This is lower than the value of 1.35 reported by $\mathrm{Lu}$ $\&$ Willmarth (1973) who used conditional sampling of hot wires. Nevertheless, this result is congruent with the hairpin vortex model where by virtue of the orientation of a hairpin vortex, Q2 ejections occur between the legs and Q4 sweeps on either side. The average length of strong individual bursts (Q2) and sweeps (Q4) (defined as a connected region of strong upwash or downwash where $-u w^{+}$is positive and greater than $2 \sigma_{-u w}^{+}$) were computed and found to be $0.04 \delta$. These lengths are comparable to the results of Lu \& Willmarth (1973).

Figure $4(b)$ shows the percentage contribution to the total Reynolds shear stress as a function of the packet length as identified by the feature extraction algorithm. The percentage contribution is computed as the ratio of total $u w$ associated with all 
the patches of a certain length to the global sum of $u w$ in all vector fields. The plot indicates that packets with lengths greater than $\delta$ contribute significantly more to the Reynolds shear stress than the smaller packets at both wall-normal positions shown. This clearly illustrates the importance of these long packets to turbulence production and transport of momentum in the logarithmic region.

The contribution of all detected patches to the Reynolds shear stress was summed in order to evaluate the percentage contribution to the mean. The percentage contribution was computed as the ratio of $u w$ associated with all the patches to the global sum of $u w$ in all the vector fields. At $z^{+}=92$, the patches were found to contribute $27 \%$ of all Reynolds shear stress even though they occupied only $4 \%$ of the total area in the vector fields. At $z^{+}=150$, the contribution was $24 \%$, and the patches occupied $4.5 \%$ of the total area. It is worth noting that the percentage contribution information is affected by the choice of the Reynolds shear stress seed threshold in the feature identification algorithm. For low thresholds $\left(0.5 \sigma_{-u w}^{+}\right)$, the contributions are as high as 55\% from a total area of $15 \%$ due to the inclusion of all possible singular vortex cores with substantial Reynolds shear stress. However, above a certain value of the threshold, i.e. $1.5 \sigma_{-u w}^{+}$, the contributions taper off towards the values quoted above. It is important to note that the patches frequently have regions of positive $u w$ in addition to the dominant negative $u w$. Hence, if only the negative values of $u w$ were included, the contribution would be even higher. The patches thus make a sizable contribution to the Reynolds shear stress considering the fact that they do not include certain Q2 events and all possible Q4 events.

\section{Summary and conclusions}

In this paper, results of stereo PIV measurements in streamwise-spanwise planes of a zero-pressure-gradient turbulent boundary layer are presented. The velocity fields and the corresponding derived gradient quantities provide a good picture of the types of structures intersecting and crossing through different heights in the logarithmic layer and beyond. An algorithm for structure identification was presented that searched for zones of uniform momentum enveloped by cores of vorticity of opposite sign that contained seed points of significant Reynolds shear stress. Some of the key conclusions from analysing the results of this algorithm and the resulting vector fields from the stereo PIV experiments are:

(i) Packets of hairpin vortices, sometimes extending over a length of $2 \delta$, were identified in the logarithmic region of the boundary layer using an objective feature extraction algorithm. This result is consistent with the conclusions of Adrian et al. $(2000 b)$ from analysing their PIV datasets in $(x, z)$-planes. Here we used stereo PIV datasets in $(x, y)$-planes which enabled us to quantify the Reynolds shear stress contribution and spanwise orientation of these structures.

(ii) The packets carry a large percentage of the Reynolds shear stress which suggests that this packet structure is an integral part of the turbulence transport mechanism. The packets contribute more than $25 \%$ to $-\overline{u w}$ even though they occupy less than $4 \%$ of the total area.

(iii) The streamwise spatial coherence and organization of vortices is seen to break down beyond the log layer, consistent with the findings of Marusic (2001) who used the attached-eddy hypothesis. The stereo PIV datasets at $z=0.2 \delta$ and $0.5 \delta$ reveal a wide range of structures but do not include long low-speed streaks caused by organization of hairpin vortices. Instead, there were regions of upwash, adjacent 
spanwise oriented strips of positive and negative wall-normal velocity and large single vortex cores.

The authors wish to thank Tim Urness, Cheonghee Lee and Professor Victoria Interrante (Computer Science) for their help with the feature extraction algorithm and visualization, and Gary Kunkel for providing his hot-wire data. We would like to thank Professor Paul Manneville for bringing the related study by Tomkins \& Adrian (2002) to our attention. This work was supported by the National Science Foundation through Grants ACI-9982274 and CTS-9983933.

\section{REFERENCES}

Adrian, R. J., Christensen, K. T. \& LiU, Z. C. $2000 a$ Analysis and interpretation of instantaneous turbulent velocity fields. Exps. Fluids 29, 275-290.

Adrian, R. J., Meinhart, C. D. \& Tomkins, C. D. $2000 b$ Vortex organization in the outer region of the turbulent boundary layer. J. Fluid Mech. 422, 1-53.

Balint, J.-L., Wallace, J. M. \& Vukoslavcevic, P. 1991 The velocity and vorticity vector fields of a turbulent boundary layer. Part 2. Statistical properties. J. Fluid Mech. 228, 53-86.

BANDYOPADHYAY, P. 1980 Large structure with a characteristic upstream interface in turbulent boundary layers. Phys. Fluids 23, 2326-2327.

BlackWELDER, R. F. \& KaPlan, R. E. 1976 On the wall structure of the turbulent boundary layer. J. Fluid Mech. 76, 89-120.

Bogard, D. G. \& Tiederman, W. G. 1986 Burst detection with single-point velocity measurements. J. Fluid Mech. 162, 389-413.

Brown, G. R. \& Thomas, A. S. W. 1977 Large structure in a turbulent boundary layer. Phys. Fluids 20, S243-S251.

Christensen, K. T. \& Adrian, R. J. 2001 Statistical evidence of hairpin vortex packets in wall turbulence. J. Fluid Mech. 431, 433-443.

Ganapathisubramani, B., Longmire, E. K. \& Marusic, I. 2002 Investigation of three dimensionality in the near field of a round jet using stereo PIV. J. Turbulence 3, 017.

Head, M. R. \& Bandyopadhyay, P. 1981 New aspects of turbulent boundary-layer structure. J. Fluid Mech. 107, 297-337.

Heist, D. K., Hanratty, T. J. \& NA, Y. 2000 Observations of the formation of streamwise vortices by rotation of arch vortices. Phys. Fluids 12, 2965-2975.

Kovasznay, L. S. G., Kibens, V. \& Blackwelder, R. F. 1970 Large-scale motion in the intermittent region of a turbulent boundary layer. J. Fluid Mech. 41, 283-326.

Longmire, E. K., Ganapathisubramani, B. \& Marusic, I. 2001 Structure identification and analysis in turbulent boundary layers by stereo PIV. In Proc. 4th Intl Symp. on Particle Image Velocimetry, Sept. 17-19, Gottingen, Germany.

Lu, S. S. \& Willmarth, W. W. 1973 Measurements of the structure of the Reynolds stress in a turbulent boundary layer. J. Fluid Mech. 60, 481-511.

Lyons, S. L., Hanratty, T. J. \& McLaughlin, J. B. 1989 Turbulence-producing eddies in viscous wall region. AIChE J. 35, 1962.

Marusic, I. 2001 On the role of large-scale structures in wall turbulence. Phys. Fluids 13, $735-743$.

Naguib, A. M. \& WARK, C. E. 1992 An investigation of wall-layer dynamics using a combined temporal filtering and correlation technique. J. Fluid Mech. 243, 541-560.

OfFEN, G. R. \& KLINE, S. J. 1975 A proposed model of the bursting process in turbulent boundary layer. J. Fluid Mech. 70, 209-228.

Robinson, S. K. 1991 Coherent motions in turbulent boundary layers. Annu. Rev. Fluid Mech. 23, 601-639.

Smith, C. R., Walker, J. D. A., Haidari, A. H. \& Soburn, U. 1991 On the dynamics of near-wall turbulence. Phil. Trans. R. Soc. Lond. A 336, 131-175.

Theodorsen, T. 1952 Mechanism of turbulence. In Proc. Second Midwestern Conf. on Fluid Mechanics, Mar. 17-19, Ohio State University, Columbus, Ohio. 
Tomkins, C. D. 2001 The structure of turbulence over smooth and rough walls. PhD thesis, Department of Theoretical and Applied Mechanics, University of Illinois at UrbanaChampaign.

Tomkins, C. D. \& Adrian, R. J. 2002 Spanwise structure and scale growth in turbulent boundary layers. TAM Rep. 1006 University of Illinois at Urbana-Champaign.

Townsend, A. A. 1976 The Structure of Turbulent Shear Flow. Cambridge University Press.

Willmarth, W. W. \& LU, S. S. 1971 Structure of the Reynolds stress near the wall. J. Fluid Mech. 55, 65-92.

Zhou, J., Adrian, R. J., Balachandar, S. \& Kendall, T. M. 1999 Mechanisms for generating coherent packets of hairpin vortices in channel flow. J. Fluid Mech. 387, 353-396. 


\section{University Library}

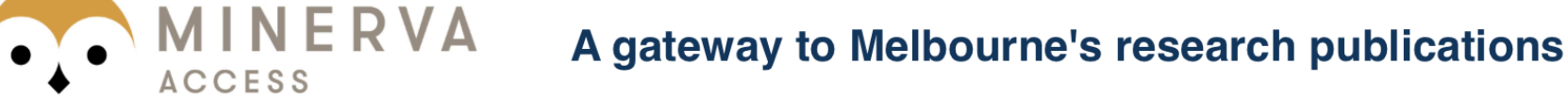

Minerva Access is the Institutional Repository of The University of Melbourne

Author/s:

Ganapathisubramani, B.;Longmire, E. K.;Marusic, I.

Title:

Characteristics of vortex packets in turbulent boundary layers

Date:

2003

Citation:

Ganapathisubramani, B., Longmire, E. K., \& Marusic, I. (2003). Characteristics of vortex packets in turbulent boundary layers. Journal of Fluid Mechanics, 478, 35-46.

Publication Status:

Published

Persistent Link:

http://hdl.handle.net/11343/34780 